\title{
Gold Nano-Island Platform for the Microfluidic Plasmonic Detection of Exosomes
}

\author{
Srinivas Bathini ${ }^{1}$, Duraichelvan Raju ${ }^{1}$, Simona Badilescu $^{1}$, Anirban Ghosh $^{2}$, Muthukumaran Packirisamy $^{1}$ \\ ${ }^{1}$ Optical-Bio Microsystems Laboratory, Department of Mechanical Industrial and Aerospace Engineering, Concordia \\ University, 1515 St. Catherine West, Montreal, Quebec, Canada, H3G 2W1 \\ s_bathin@encs.concordia.ca; d_raj@ encs.concordia.ca; simonabadilescu0@gmail.com; mpackir@encs.concordia.ca \\ ${ }^{2}$ Atlantic Cancer Research Institute \\ 35, rue Providence Street, Pavilion Hotel-Dieu Pavilion, Moncton, New Brunswick, Canada, E1C 8X3 \\ AnirbanG@canceratl.ca
}

\section{Extended Abstract}

Noble metals (gold and silver) possess interesting plasmonic properties, allowing the detection at the nano-scale is by observing their LSPR band shift, due to binding events and change of the refractive index [1-2]. A shift towards the longer wavelength of the local LSPR band is noticed upon the binding of bio-molecules onto the metal nanoparticles immobilized on a substrate. While a great number of publications exist on the LSPR phenomenon and its applications, there are only a few trying to integrate LSPR biosensors and microfluidics [3-4]. Recently microfluidics-based designs have been developed and implemented by several researchers in order to isolate and quantify biomolecules which are bio markers for the early diagnosis of cancer. Exosomes are the carriers of genetical and molecular information from parent to distal cells [5]. At present, the commonly used protocols for the isolation and detections of exosomes are the high-speed centrifugation and ultracentrifugation which are time consuming, with relatively low yield. Therefore, a versatile and efficient technique is required to for the isolation and detections of exosomes for further diagnosis at clinical level.

The objective of the present work is the plasmonic detection of exosomes based on the sensitivity of the Au LSPR band to binding events. As mentioned earlier, the sensing mechanism makes use of the change in the position of the Au LSPR band due to the binding of the chemical entities and biomolecules to the immobilized gold nano-island, resulting in a shift of the band towards longer wavelengths. For the capture and detection of exosomes, in the present study, multilayers of gold $\mathrm{Au}$ ) nanoparticles were deposited by convective assembly from the gold colloidal suspension [4]. The glass substrates were further annealed at $560^{\circ} \mathrm{C}$ in order to modify the morphology from the multilayers of gold nanoparticles aggregates to nano-islands. In order to capture the exosomes, the affinity based synthetic polypeptide called Venceremin, Vn96 is used [6]. This molecule is specifically designed and validated to capture exosomes, having a strong affinity towards the canonical heat shock proteins (HSP), present on the surface of exosomes.

The detection method is initially carried out at the substrate level, by performing the sensing protocol in a discontinuous manner. The concentrations of the chemical and biological entities at each stage of biosensing were optimized in order to facilitate the transfer to the microfluidics stage. The results indicated that this platform, is promising for the detection of exosomes. Further, in order to enhance the sensitivity of the detection and to accomplish the molecular profiling of the captured exosomes, microfluidic devices were designed, developed and tested.

The results indicated that label-free technique, based on the sensitivity of the Au-LSPR band to the surrounding environment is promising for the detection of MCF-7 exosomes by the affinity approach, using the Vn96 polypeptide. This approach seems to be more sensitive than the more traditional immunosensing method. The biosensing protocol was transferred successfully to the microfluidic environment for the detection and capture of exosomes.

\section{References}

[1] K. S. Lee, and M. A. El-Sayed, "Gold and silver nanoparticles in sensing and imaging: sensitivity of plasmon response to size, shape, and metal composition," J Phys Chem., vol 110, pp. 19220-19225, 2006.

[2] T. Chung, S. Y. Lee, E. Y. Song, H. Chun and B. Lee, "Plasmonic nanostructures for nano-scale bio-sensing," Sensors, vol.11, no.11, pp. 10907-10929, 2011. 
[3] C. Huang, K. Bonroy, G. Reeckmanns, W. Laureyn, K. Verhaegen, I. de Vlaminck, L. Lagae, G. Borghs, "Localized surface plasmon resonance integrated with microfluidic chip," Biomed Microdevices, vol. 11, pp. 893-901, 2009.

[4] J. Ozhikandathil, S. Badilescu, M. Packirisamy, "Gold nanoisland structures integrated in a lab-on-a-chip for plasmonic detection of bovine growth hormone," Journal of Biomedical Optics, vol. 17, no.7, p. 077001, 2012.

[5] G. Fuhrmann, I. K. Herrmann, M. M. Stevensa, "Cell-derived vesicles for drug therapy and diagnostics: Opportunities and challenges," Nano Today, vol. 10, pp. 397-409, 2015.

[6] A. Ghosh, M. Davey, I. C. Chute, S. G. Griffiths, S. Lewis, et al., "Rapid Isolation of Extracellular Vesicles from Cell Culture and Biological Fluids Using a Synthetic Peptide with Specific Affinity for Heat Shock Proteins," PLoS ONE, vol. 9, no. 10, 2014. 